Service social

\title{
Projet "Chômeurs Créateurs Associés ", par Marie-Chantal Guédon, Saint-Jean-sur-Richelieu, Centre de formation à l'Autogestion du Haut-Richelieu (C.P. 532, Saint-Jean-sur-Richelieu, Québec, J3B 6Z4), 1989, 84 pages.
}

\section{Gérald Doré}

Volume 39, numéro 2, 1990

Les problèmes sociaux

URI : https://id.erudit.org/iderudit/706495ar

DOI : https://doi.org/10.7202/706495ar

Aller au sommaire du numéro

Éditeur(s)

École de service social de l'Université Laval

ISSN

1708-1734 (numérique)

Découvrir la revue

Citer ce compte rendu

Doré, G. (1990). Compte rendu de [Projet "Chômeurs Créateurs Associés », par

Marie-Chantal Guédon, Saint-Jean-sur-Richelieu, Centre de formation à

l'Autogestion du Haut-Richelieu (C.P. 532, Saint-Jean-sur-Richelieu, Québec, J3B

6Z4), 1989, 84 pages.] Service social, 39(2), 252-254.

https://doi.org/10.7202/706495ar d'utilisation que vous pouvez consulter en ligne.

https://apropos.erudit.org/fr/usagers/politique-dutilisation/ 
dement, de culte, de production et échange " pour expliquer l'existence du lien constant entre la prison et la pauvreté.

L'utilisation de la méthode historique est à notre avis un procédé judicieux et original. En même temps que l'auteur fournit une source crédible de références, son œuvre prend l'allure d'un récit qui peut intéresser même les néophytes du domaine de la criminologie. Le vocabulaire choisi est simple, le style est clair, l'écriture est sans ambiguïté, la démarche est logique et convaincante. Le caractère d'impression, serré, est toutefois à déplorer n'étant pas de nature à faciliter la lecture du volume.

Par les aspects historiques soulevés, l'ouvrage suscitera certainement de l'intérêt chez les professionnels et ex-professionnels du milieu carcéral, mais peut-être aussi un certain malaise ou un requestionnement critique parce que le volume révèle des manigances exercées bien souvent à leur insu ou en l'absence de connaissances suffisantes.

La force véritable de ce volume réside dans le fait qu'il met à jour, pour les présentes générations de professionnels des services correctionnels, les enjeux qui ont originé et qui persistent dans le système de justice pénale québécoise. Dans ce sens, l'ouvrage du professeur Laplante constitue un outil remarquable d'enseignement et de formation en matière de pénologie et de politique criminelle au Québec. Tout lecteur est incité à développer un questionnement critique et conscient sur le sens et la portée de la prison comme institution. Si tel était le but de l'auteur, l'objectif est atteint.

La dernière portion de la troisième partie du volume, titrée "la prison au cœur du social », nous interroge par rapport au reste de l'ouvrage. En dépit de restrictions annoncées, on ne sait trop s'il s'agit d'une fin mal bouclée, de l'amorce d'une conclusion qui reste inachevée ou d'une stratégie de l'auteur qui veut inciter le lecteur à se positionner. L'impression qui en reste est floue et donne malheureusement à penser que l'auteur a manqué de souffle pour conclure, ce qui enlève un peu de fini au volume qui se présentait jusqu'ici comme un travail remarquable.

Outre cette dernière remarque de portée mineure, il s'agit d'un excellent ouvrage critique sur le système de justice en contexte québécois, à recommander pour lecture et usage à des fins d'enseignement.

Germain Trottier

École de service social

Université Laval

\section{Projet "Chômeurs Créateurs Associés ", par Marie-Chantal} GUÉDON, Saint-Jean-sur-Richelieu, Centre de formation à I'Autogestion du Haut-Richelieu (C.P. 532, Saint-Jean-surRichelieu, Québec, J3B 6Z4), 1989, 84 pages.

Cette monographie décrit et évalue une intervention menée entre février 1986 et octobre 1988, auprès de jeunes sans emploi, de 18 à 30 ans, de la région de Saint-Jean-sur-Richelieu. L'approche retenue était celle du "chômage créateur ", défini comme une " attitude consistant à dépasser l'attente passive ou la recherche effrénée d'un emploi pour exploiter le temps libéré par l'absence d'emploi et le remplir par des activités ou des moments de réflexion susceptibles de favoriser un meilleur épanouissement et une plus grande prise 
en charge de sa vie» (p. 4). Cette approche s'inscrit dans le courant des approches dites " intégrées ", dans la mesure où elle visait à couvrir quatre composantes vues comme "formant une séquence": individu, groupe, économique, communautaire. Dans les termes mêmes des concepteurs de l'approche, " on prévoyait que les individus exploreraient d'abord leur situation personnelle, puis se mobiliseraient autour de petits projets de groupes, dont certains à caractère économique, dans un processus de prise en charge collective se prolongeant au niveau de la communauté locale, avec le soutien de celle-ci » (p. 6). En réalité, il en fut autrement et l'auteure en rend compte avec beaucoup de rigueur et une grande honnêteté intellectuelle. Elle nous trace une juste évaluation de l'expérience, évitant tout autant l'écueil de I'autojustification après coup que celui de l'accablement démobilisant. Les informations sont claires et précises, les jugements sont fondés sur une argumentation serrée, de sorte que se dégagent de cet ouvrage des perspectives susceptibles d'orienter judicieusement l'intervention sociale avec les chômeurs et chômeuses.

Le texte est divisé en neuf chapitres couvrant le projet et son contexte (et contenant notamment une formulation concise de la problématique et de l'approche), le déroulement général de la réalisation du projet, les caractéristiques de la clientèle rejointe, les activités faites avec la clientèle, les modes de participation de la clientèle cible, ce qu'en pensent les participants, l'impact du projet dans le milieu, le bilan et les perspectives.

"L'ambiguïté qui entoure la notion de chômage créateur »se fit jour dès le début du projet et cette constatation est mise en évidence avec beaucoup de franchise. "Devait-on travailler essentiellement sur l'utilisation du temps de non-emploi, souligne l'auteure, en vue de l'épanouissement personnel vers une plus grande autonomie, ou fallait-il viser le retour au travail et faciliter celui-ci... C'est surtout par rapport au volet " économique » du projet, ajoutet-elle, que les difficultés se firent sentir. Comment pouvait-on favoriser la mise sur pied d'entreprises (donc de lieux d'emplois) dans le cadre d'un programme axé sur I'utilisation positive du non-emploi ?»(p. 10). Comme le dira un participant, au moment de l'évaluation, "la notion de chômage créateur... c'était acceptable pendant un certain temps, mais [...] il fallait à un moment donné penser aux choses sérieuses, c'est-à-dire au travail " (p. 62). Le mode de recrutement adopté (un programme de travaux temporaires) n'allait guère contribuer à dissiper cette ambiguïté. "Venus pour des " jobines ", beaucoup de participants ne semblaient pas intéressés à s'engager dans autre chose sinon des activités récréatives peu exigeantes $($ p. 16). Les correctifs apportés dans la dernière étape du projet, avec un recrutement axé sur des activités structurées (vacances familiales et coopérative de récupération), ont d'ailleurs permis de constater que "si c'était cette dernière stratégie qui avait d'abord été utilisée, on aurait probablement eu un bilan global assez différent » (p. 16).

Comme il arrive malheureusement trop souvent avec les approches dites " intégrées", il est résulté des choix stratégiques effectués " que le volet "psycho-individuel " a été beaucoup plus touché que les autres, suivi par le volet "psycho-social" (p. 34). La portée de la constatation est ici malheureusement atténuée par un effort d'optimisme banalisant qui tranche avec le ton de l'ensemble de l'ouvrage : " peu importe, par exemple, que les projets des participants et participantes aient abouti ou non. Le plus important, c'est qu'ils aient été entrepris... [etc.]" (p. 40). Le chapitre "bilan et perspectives" renoue heureusement sur ce point avec la rigueur de l'ensemble de l'exposé. On y fait état d'un triple glissement survenu entre le projet et sa réalisation : "du collectif vers l'individuel, du socio-économique 
vers le psychologique et du didactique vers le ludique » (p. 73). Plus important encore, on y effectue un retour sur la notion du chômage créateur et on y propose des perspectives de relance, avec des vues précises par rapport à certains paramètres tels que la clientèle, les objectifs d'intervention, le milieu d'intervention, le cadre d'intervention, l'équipe d'intervention, le contenu du programme, etc. Certains lieux communs y sont enfin démystifiés, tels le développement de "l'employabilité " et l'incitation à la création d'entreprises. Un bilan donc d'une belle facture et d'une grande qualité. Je n'aurais qu'un reproche à lui adresser: que la dynamique de la relation vécue entre intervenants-intervenantes et participants-participantes ait été délibérément mise de côté (p. 52-53). C'est la dimension qu'il resterait à explorer pour une vue complète de la relance à effectuer.

Une version abrégée de ce rapport d'évaluation est disponible sous la description bibliographique suivante : Vivre malgré l'absence d'emploi : le chômage créateur, par Michel Boyer, Marie-Chantal Guédon et Anne Kettenbeil, Saint-Jean-sur-Richelieu, Centre de formation à l'Autogestion du HautRichelieu, 1989, 24 pages. Je recommande la lecture de la version intégrale pour une pleine compréhension de la nature et de la portée de l'intervention réalisée.

Gérald Doré

École de service social

Université Laval

\section{Psychiatrie clinique, approche bio-psycho-sociale, par Pierre LALONDE, Frédéric GRUNBERG et collaborateurs, Gaëtan Morin éditeur, $2^{\mathrm{e}}$ édition, 1988, 1348 pages (distribué en Europe et en Afrique par les éditions Eska).}

"Une psychiatrie qui continue d'être française et européenne tout en s'inspirant au maximum des travaux des écoles américaines "nous dit-on dans la préface. II s'agit d'une tentative intéressante de synthèse, à partir de notre situation géographique et politique particulière, et d'intégration des théories multiples qui influencent la compréhension des comportements humains, normaux et psychopathologiques.

Ce livre est clair et précis, de lecture agréable et facile. II faut rendre hommage à ce souci de clarté et à l'évitement systématique d'un vocabulaire obscur et hermétique. L'impression est nette, la mise en page soignée et variée, le tout bien aéré. Un produit final qui met en appétit de lecture !

L'ouvrage fait appel à cinquante-quatre collaborateurs et est divisé en six parties. On y traite des sujets suivants : introduction à la psychiatrie, état actuel de la psychiatrie, relation médecin et malade, épidémiologie, psychophysiologie, développement de la personnalité, examen psychiatrique, syndromes cliniques psychiatriques (des troubles anxieux, en passant par l'alcoolisme et les urgences), troubles de la sexualité, aspects spécifiques de la psychiatrie (femme-famille-couple), pédopsychiatrie (de l'évaluation aux stades de développement) et enfin, traitements psychiatriques divisés en trois parties : traitements pharmacologiques, psychothérapies et thérapies psychophysiologiques. Une dernière partie aborde plus spécifiquement les aspects administratifs et légaux. 